\title{
El Paciente con Hemorragia y Tiempo de Sangría Prolongado: Clasificación y Diagnóstico Diferencial
}

\author{
Dra. Esperanza Marzouka B.1 * Dr. Ernesto Ríos L. I; Dr. Diego Mezzano A.2 ; Dra. Waltraud Schuh 0.1: \\ B.Q. Eduardo Aranda L. ${ }^{2}$; T.M. Cecilia Cataldo T.1; T.M. Eugenia Legües S. 2.
}

\section{Factor VIII and Platelet Aggregation in Patients with Hemorrhagic Symtoms and Prolonged Bkeding Time}

\begin{abstract}
Seventy six patients with hemorthagic symtoms and prolonged template bleeding time were studied for Factor VIII (F. VIII) Complex components (F. VIII: C., F. VIIL R.: Ag., F. VIll: $C O R$ and bidirectional inmune electrophoresis) and platelet aggregation. Among 66 patients which completed the laboratory work-up $\$ 1$ (77\%) were finally classified as Von Willebrand's Disease (V.W.D.); 45 corresponded to type I VWD and 6 to type II. Four patients were demonstrated to have congenital platelet abnomalities consisting in Storage Pool disease (n $=2)$ and Glanzman' disease $(n=2)$. Three patients showed evidence of mild A type Hemophilia, one of Ehlers-Danlos's Syindrome and in seven it was not possible to achieve a definite diagnosis.

(Key mords: Factor Vill. Platelet aggregation. Prolonged bleeding time. Platelet abnormalities, Von Willebrands's disease).
\end{abstract}

La enfermedad de von Willebrand (E.v.W.) es un trastorno hereditario de la hemostásis, caracterizado por hemorragia de piel y mucosas y por un estudio de laboratorio con tiempo de sangria prolongado, disminución de la adhesividad plaquetaria y menor actividad procoagulante del factor VIII.

En los últimos años se ha avanzado enormemente en el estudio de la estructura, biología $y$ función del factor VHII $y$ se han desarrollado nuevos métodos de laboratorio, que han permitido el diagnóstico de las formas leves y moderadas de la enfermedad, como tambièn una adecuada clasificación de ella.

Actualmente se postula que el factor VIII es un complejo molecular de dos componentes ${ }^{2-2 \cdot 3}$. El primero es una unidad coagulante de bajo peso molecular (F. VIII: C), que participa como cofactor en la vía intrinseca de la coagu. lación, se hereda ligado al cromosoma $X$, está ausente o disminuído en la hemofilia $A$ y leve 0 moderadamente disminuido en la E.v.W. El segundo componente es la unidad antigénica $(F$. VIII R.: Ag) presente en el plasma en forma de multímeros de alto peso molecular $\left(8 \times 10^{5}\right.$ a 20

I. Unidad de Hematología, Hospital Luis Caivo Mackenna, Facultad de Medicina, División Ciencias Médicas Oriente, Universidad de Chite.

2. Departamento de Hernatología, Oncología y Banco de Sangre, Facultad de Medicina, Universidad Católica de Chile.

* Dirección actuali Instituto de Salud. $x 10^{6}$ daltons) que se hereda en forma autosómica: estos polímeros actúan como puente entre las plaquetas y el subendotelio vascular, permitiendo la adhesividad plaquetaria; además estabilizan y estimulan la síntesis del F. VIll: C. Por ello, la disminución del F. VIII R.: Ag. se acompaña de una disminución del F. VIII: C.

En la E.y.W. hay un defecto del segundo componente el F. VIII R.: Ag. Este se puede cuantificar por inmunoelectroforesis y también midiendo la actividad co-factor ristocetina $(\mathrm{F}$. VIII: CoR), que estudia la capacidad de las plaquetas normales de aglutinarse en presencia de ristocetina.

Ruggeri y colaboradores ${ }^{4}$ han clasificado a estos pacientes en dos grandes grupos:

El Tipo I se caracteriza por una reducción cuantitativa de las actividades del complejo VIII. El Tipo II tiene un defecto cualitativo, con polimerización deficiente del complejo VIII, detectable por inmunoelectroforesis bidireccional de F. VIII (IEFB-VIII).

En los últimos años se ha avanzado también en el estudio de los defectos de la función plaquetaria $^{5.7}$, que han sido calificados en defectos de adhesion (Enfermedad de Bernard Soulier), de agregación (Enfermedad de Glanzman, afibrinogemia) y de la secreción (Enfermedad del compartimiento de depósito o "Storage Pool Disease"). Estos trastornos se investigan con el tiempo de sangría y el estudio de la agregación plaquetaria.

Con estos antecedentes, que muestran el 
avance en el estudio de estas enfermedades, nos interesamos por dar mayor precisión al diagnóstico de 76 pacientes que consultaron con manifestaciones hemorrágicas y tiempo de sangría prolongado.

\section{MATERIAL Y METODO}

Se estudiaron 76 niños cuyas edades fluctuaron entre 1 y 15 años con antecedentes hemorrígicos personales, con o sin antecedentes familiares, entre los que destacaban, por su mayor frecuencia, epistaxis $y$ equimosis espontáneas o secundarias a traumatismos, con menor frecuencia hemorragias secundarias a extracciones dentarias $o$ a intervenciones quirúrgicas, metrorragia, hematomas, petequias y en un caso hematuria, que cumpliesen, además, los siguientes requisitos de laboratorio de orientación en coagulación:

Tiempo de sangría de Ivy modificado prolongado, sin ingestión de ningún medicamento en los 10 días previos. Es considerado normal un valor hasta 7,75 min. ${ }^{8}$ Recuento de plaquetas normal, hecho en cámara con contraste de fase ${ }^{9}$. Tiempo de tromboplastina parcial activado, normal o prolongado. Tiempo de protrombina normal.

Una vez incluídos en el estudio, se efectuaron en todos los pacientes una nueva medición del tiempo de sangría de Ivy modificado y estudio del complejo VIII.

La actividad procoagulante del complejo VIII (F. VIII: C) se determinó por el método en una etapa $^{10}$, usando sustrato DADE; F. VIII R.: Ag se cuantificó por electroinmunoensayo mediante la técnica de Laurell11 ${ }^{11}$ adaptada por Zimmerman $^{12}$. La actividad cofactor ristocetina ( $F$. VIII: $\mathrm{Co}-\mathrm{R}$ ) se determinô por microtécnica de aglutinación en placa ${ }^{13}$.

En los pacientes con tiempos de sangría prolongados, pero con resultados normales para el complejo VIII, se continuó el estudio, realizán. dose uno o más de los siguientes exámenes:

Agregación plaquetaria en plasma rico en plaquetas con A.D.P., epinefrina, colágeno y araquidonato de sodio, usando un agregómetro Chrono-Log ${ }^{14}$. Inmunoelectroforésis bidireccional del F. VIII (I.E.F.B. - VIII) ${ }^{15}$. Repetición del estudio del complejo VIIIl.

\section{RESULTADOS}

De los 76 pacientes que ingresaron al estudio, 66 lo completaron. Los resultados de los exáme. nes del laboratorio, permitieron agruparlos en 4 grupos de enfermedades (Figura 1).

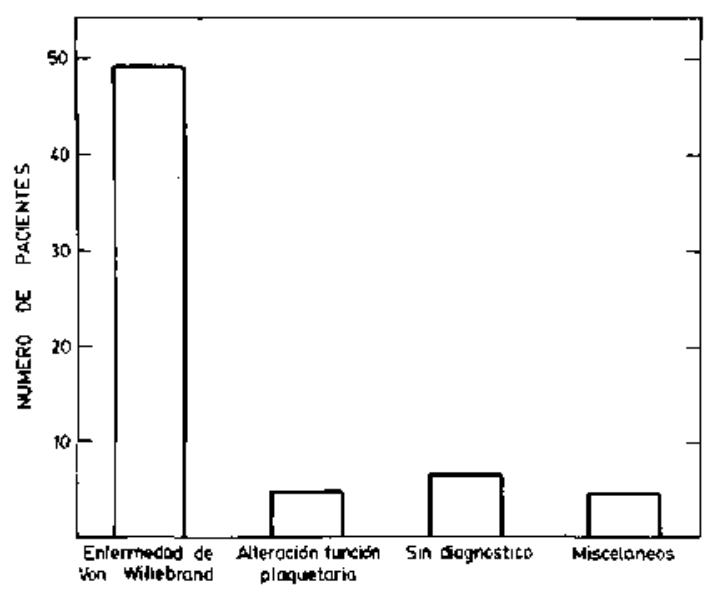

Figura 1. Diagnóstico en pacientes con antecedentes hemorrágicos y tiempo de sangria de Jvy modificado prolongado.

En 51 pacientes se diagnosticó Enfermedad de von Willebrand. De ellos, 45 pacientes corresponden a E.v.W. Tipo I. El diagnóstico se basó en los tiempos de sangría y en el estudio del complejo VII, analizándose el número de parámetros de laboratorio alterados (tiempo de sangria, F. VIII: C, F. VIIl R.: Ag, F. VIII: Cor). Los pacientes con dos o más exámenes de laboratorio anorma. les o 1 examen alterado, más el antecedente de un familiar con la enfermedad, se catalogaron como E.v.W. Tipo I. El análisis individual de los pacientes se muestra en las Tablas 1 y 2 , destacando que 20 enfermos tuvieron 3 ó 4 parámetros de laboratorio alterados, 21 enfermos tuvieron 2 patámetros de laboratorio anormales y sólo 4 enfermos presentaron un solo examen alterado más el antecedente de un familiar con la enfermedad. Los restantes 6 niños (Tabla 3) pertenecen al Tipo II de la E.v.W. EJ diagnóstico, en 5 de ellos se basó en I.E.F.B. VIII alterada, con movilidad anódica excesiva del F. VIII R.: Ag y en 1 ó más de las otras pruebas alteradas. El sexto paciente de este grupo tuvo una I.E.F. B. VIII normal, pero presentaba síntomas hemorrá. gicos, un tiempo de sangría prolongado y tiene 2 hermanos en este mismo subgrupo.

En 4 pacientes se encontró un defecto congénito plaquetario; 2 presentaban malformaciones de las extremidades, recuentos de plaquetas entre 50 y $150 \mathrm{mil} \mathrm{x} \mathrm{mm}^{3}$ y tiempos de sangria entre 9 y 30 minutos, desproporcionadamente prolongados con respecto a la trombocitopenia. Los exámenes revelaron funciones normales de $\mathrm{F}$. VIII y agregación plaquetaria alterada en presencia de adrenalina, A.D.P. y colágeno, pero normal con araquidonato de sodio. Con estos 
antecedentes se diagnosticó Enfermedad del compartimento de depósito. Los otros 2 pacientes de este grupo tenían tiempos de sangria de 14 minutos y de más de 20 minutos respectivamente, el estudio del Complejo VIII fue normal y no hubo agregación plaquetaria con A.D.P., adrena. lina, colágeno, araquidonato de sodio e ionóforo. En ellos se confirmó la existencia de tromboastenia o Enfermedad de Glanzman.

Tabla 1.

linfermedad von Willebrand Tipo I:

2 Parámetros de Laboratorio Altesados

\begin{tabular}{|c|c|c|c|c|}
\hline & $\begin{array}{c}\text { Sangría } \\
\text { (minutos) }\end{array}$ & F. VIIIc & $\begin{array}{r}\text { E. vill R: } \\
(\mu ; m l)\end{array}$ & $\begin{array}{c}A g F \\
(\%)\end{array}$ \\
\hline \multirow[t]{2}{*}{ 1. A.C.V. } & 10 & 67 & 0.80 & 72 \\
\hline & 8 & 90 & 0.77 & 54 \\
\hline 2. B.I.R. & 8.5 & 98 & 0.68 & 55 \\
\hline 3. D.C.C. & [] & 100 & l & 76 \\
\hline 4. E, M.A. & 14 & 28 & 1.24 & $>100$ \\
\hline S. IN.A. & 9.5 & 90 & 0.96 & 55 \\
\hline 6. H.O.S. & 10 & 100 & 05 & 100 \\
\hline 7. CM.M. ${ }^{\circ}$ & 7.5 & 46 & 0.75 & 50 \\
\hline 8. A.S.U. & 15.5 & $\begin{array}{l}42 \\
42\end{array}$ & $\begin{array}{l}0.99 \\
1 .\end{array}$ & $\begin{array}{l}95 \\
90\end{array}$ \\
\hline 9. A.P.M. & 11.5 & 90 & 0.52 & 77 \\
\hline 10. В.Р.М. ${ }^{\circ}$ & 5.5 & 100 & 0.52 & 80 \\
\hline \multirow[t]{2}{*}{ 11. A.S.M. } & 18 & 100 & 0.76 & 50 \\
\hline & 10 & 100 & 1.08 & 33 \\
\hline 12. S.T.E. & 13.5 & 62 & 0.54 & 100 \\
\hline \multirow[t]{2}{*}{ 13. E.S.M } & 8 & 100 & 0.88 & 58 \\
\hline & 9.5 & 100 & 1.1 & 100 \\
\hline 14. M.P.B. & 9 & 25 & 1.1 & 75 \\
\hline \multirow[t]{2}{*}{ 15. V.A.C. } & 9.5 & 100 & 0.88 & 80 \\
\hline & & 62 & 0.6 & 65 \\
\hline 16. R.G.R. & 14 & 15 & 0.95 & 65 \\
\hline 17. A.C.C. & 17 & 80 & 0.72 & 75 \\
\hline 18. S.H.B. & $\begin{array}{r}10 \\
6\end{array}$ & 92 & 0.64 & 42 \\
\hline 19. C.P.M. & $\begin{array}{r}12.5 \\
7.5\end{array}$ & 100 & 0.96 & 33 \\
\hline 20. L.T.E. & $\begin{array}{l}8.5 \\
5\end{array}$ & 89 & 1.1 & 51 \\
\hline 21. V.D.S. & $\begin{array}{r}11.5 \\
4.5\end{array}$ & 100 & 0.75 & 75 \\
\hline
\end{tabular}

Valores normates: $\begin{array}{llll}7.75 & 60-150 \quad 0.62-1.5 & >60 \%\end{array}$

${ }^{0}=$ Familiar de 2 pacientes con E.v. $W$.

En otros 4 pacientes se encontraron diferentes alteraciones de la coagulación; 3 tenían tiempos de sangría levemente prolongados, actividad disminuída de F. VIII:C y el resto del estudio normal, confirmándose Hemofilia A leve. El cuarto paciente presentó tiempos de sangría prolongados, pero el estudio de funciones de F. VIll y la agregación plaquetaria fueron normales; en él se diagnosticó un síndrome de Ehlers-Danlos.

En 7 niños, a pesar del estudio repetido, no se logró un diagnóstico definitivo.

De los 10 pacientes excluídos del análisis de los resultados 6 presentaban sólo prolongación del tiempo de sangría y al reevaluar sus antecedentes hemorrágicos personales $y$ familiares se encontró que estos eran pobres y sus nuevos tiempos de sangría fueron normales. Los 4 niños restantes no pudieron ser bien evaluados, ya que, a pesar de tener todos dos o más tiempos de sangria prolongados, antecedentes hemorrágicos personales, familiares o ambos, mostraron actividades normales de Complejo VIIl y agregación plaquetaria, sin embargo, no pudo efectuarse en ellos I.E.F.B. - VIII ni repetir el estudio del Complejo VIIl para lograr un diagnóstico definitivo.

Tabla 2.

Enfermedad von Willebrand Tipo I: 3 ó 4 Parámetros de Laboratorio Alterados

\begin{tabular}{|c|c|c|c|c|}
\hline & $\underset{\text { (minutas) }}{\text { Sangría }}$ & $\underset{\substack{\text { F. Vill } \\
\text { fic }}}{ }$ & $\begin{array}{l}\text { I:. VIII R: Ag } \\
(\mu / \mathrm{ml})\end{array}$ & F. V[][ Cor \\
\hline 1. D.G.C. & 20.5 & 24 & 0.25 & $<10$ \\
\hline 2. G.G.C. & $>25$ & 25 & 0.25 & $<10$ \\
\hline 3. L.C.A. & 9 & 50 & 0.25 & 29 \\
\hline 4. G.L.P. & 13.5 & 51 & 0.5 & 12.5 \\
\hline 5. D.P.J. & 12 & 45 & 0.6 & 39 \\
\hline 6. G.S.I. & $>25$ & 56 & 0.14 & 5 \\
\hline 7. E.H.R. & 23.5 & 75 & 0.32 & 36 \\
\hline 8. S.B.R. & 4.5 & 30 & 0.22 & 4 \\
\hline \multirow[t]{2}{*}{ 9. V.M.A. } & 15 & 43 & 0.46 & 70 \\
\hline & 15 & 20 & 0.75 & 65 \\
\hline 10. A.T.Y. & 12 & 29 & 0.60 & 100 \\
\hline 11 . R.V.F. & 16.5 & 15 & 0.57 & 90 \\
\hline \multirow[t]{3}{*}{ 12. S.P.E. } & 12 & & & \\
\hline & 5.5 & 85 & 0.46 & 60 \\
\hline & 8 & 100 & 0.8 & 90 \\
\hline 13. G.J.T. & 12 & 55 & 0.6 & 75 \\
\hline \multirow[t]{2}{*}{ 14. A.C.G. } & 9.5 & 70 & 0.88 & 100 \\
\hline & 4 & 33 & 0.44 & 36 \\
\hline \multirow{2}{*}{$\begin{array}{l}\text { 15. C.V.C. } \\
\text { 16. A.D.M. }\end{array}$} & $1 d$ & 43 & 0.75 & 60 \\
\hline & 6.5 & 48 & 0.81 & 40 \\
\hline \multirow[t]{2}{*}{ 17. 1.0.M. } & 8 & & & \\
\hline & 6.5 & 48 & 0.92 & 52 \\
\hline \multirow[t]{2}{*}{ 18. H.G.R. } & 12.5 & & & \\
\hline & 2.5 & $\$ 5$ & 0.5 & 80 \\
\hline \multirow[t]{2}{*}{ 9. S.C.R. } & 8.5 & & & \\
\hline & 6.5 & 30 & 0.75 & 80 \\
\hline 20. M.B.P. & $\begin{array}{c}17.5 \\
7\end{array}$ & 100 & 0.37 & 6 \\
\hline Vajorcs normales: & 7.75 & $60-150$ & $0.62-1.5$ & $>60 \%$ \\
\hline
\end{tabular}

Tabla 3.

Enfermedad von Willebrand - Tipo II

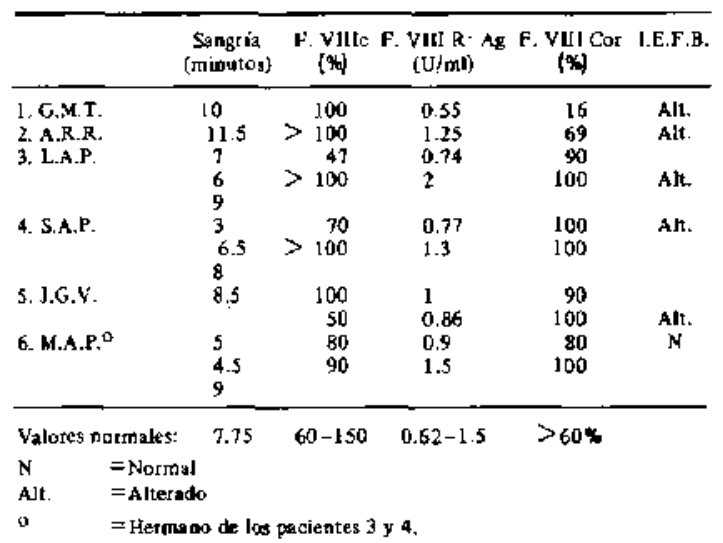




\section{DISCUSION}

La mayoría de los pacientes estudiados $(77 \%)$ corresponde a la E.y.W. De èstos, $88 \%$ pertenecian al Tipo I de la enfermedad, con alteración cuantitativa del Complejo VIII y sólo $12 \%$ aI Tipo II, con defecto cualitativo, detectable por I.E.F.B. - VIII. Esto concuerda con publicaciones del extranjero que señalan predominio de pacientes con E.v.W. Tipo I ${ }^{26}$. En este grupo de enfermos no se observaron diferencias por sexo, hecho también comprobado en otras publicacio. nes.

El estudio de estos pacientes confirmó una amplia gama de variaciones entre ellos, no sólo en los resultados de laboratorio, sino también en las manifestaciones clínicas: Algunos enfermos tjenen muy pocos síntomas y requieren estudios repetidos, para confirmar la E.v.W., y otros tienen muchos síntomas: entre ambos extremos hay una amplia gama de expresiones clínicas y de laboratorio. En la Figura 2 se observa claramente que algunos pacientes tienen compromiso de 3 ó 4 parámetros de laboratorio, ellos corresponden en general, aunque no siempre, a los pacientes con más síntomas en los que la enfermedad es más aparente. En.cambio, los enfermos con un parámetro de laboratorio alterado y un familiar con la enfermedad o los pacientes con 2 parámetros de laboratorio alterados, son en general menos sintomáticos y el diagnóstico es más difícil.

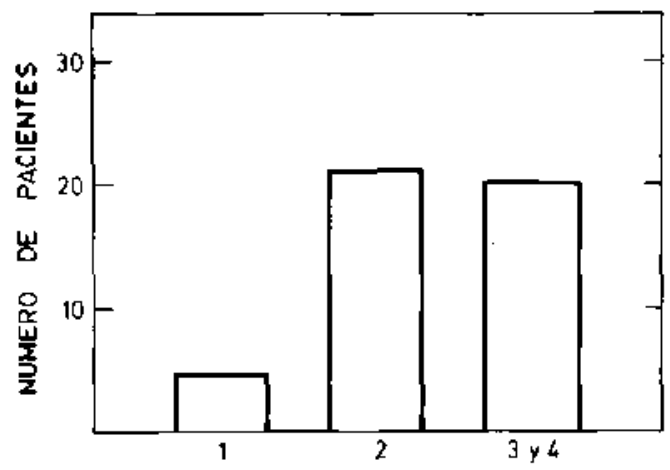

NUMERO DE PARAMETROS DE LABORATORIO ALTERADOS

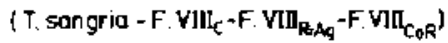

Figura 2. Fafermedad de von Willebrand tipo I: Frecuencin de número de parámetros de laboratorio alterados.

Las variaciones descritas también se observan en un mismo individuo estudiado en diferentes momentos (Tabla 1 y 2), lo que justifica, en pacientes con manifestacjones hemorrágicas, repetir los exámenes para llegar al diagnóstico.
En 7 pacientes, a pesar de tener antecedentes hemorrágicos personales y familiares importantes y dos o más tiempos de sangría prolongados, con el resto de los exámenes normales, incluyendo el Complejo VII, I.E.F.B. - VIII y la agregación plaquetaria, no se pudo llegar a un diagnóstico definitivo. Este grupo puede corresponder a trastornos de hemostasia no identificables con los métodos actuales y aún corresponder a una Enfermedad de Bernard Soulier.

\section{RESUMEN}

Publicaciones recientes informan de una alta frecuencia de la Enfermedad de von Willebrand (E.v.W.) en pacientes con tiempo de sangria prolongado y recuento de plaquetas normal, destacando el uso de nuevos metodos de laboratorio que permiten el diagnóstico de los grados leves y moderados de la enfermedad.

Se estudiaron 76 pacientes pediátricos con antecedentes hemorrágicos y al menos un tiempo de sangría de Ivy prolongado, sin ingestión previa de medicamentos. De ellos, 66 completaton el estudio, pudiendo ser clasificados en las siguientes afecciones:

Enfermedad de von Willebrand, 51 pacientes, trastorno congénito de la función plaquetaria, 4 , otros trastornos de la coagulación, 4 no se logró hacer un diagnóstico definitivo en 7 pacientes.

\section{REFERENCIAS}

1. Hoyer, L.W: "The Factor VIII Complex, Structure and Function". Blood 58: 1, 1981.

2. Bloom, A.L.: "The Biosynthesis of Factor VIII", Clin. Haematol. 8: 53, 1979.

3. Zimmerman, T.S. and Ruggeri, Z.M.: "Von Willebrand's disease". Clin. Haematol. 12: 135, 1983.

4. Ruggeri, Z.M. Pareti, F.I, Mannucci, P.M, Cinarello, N. Zimmerman, T.S.: "Heightened Interaction Bciween Platelets and Factor VIIl / von Willebrand's Factor in a New Subtipe of yon Willebrand's Disease". N. Engl. J. Med. 302: 1047, 1980

5. Shattil, S.I., Bennett, J.S.: "Platelets and their Nembranes in hemostasis: physiology and Pathophysiology. Ann. Intern. Med. 94: 108, 1980.

5. Weiss, H.J.: "Congenital disorders of Platclet function". Sem. Hematol. 17: 228, 1980.

7. Vermylen, J., Vadenhorst, P.N. Deckmyn, H. and Arnout. J.: "Normal Mechanism of Platelet Function". Clin. Haematol. 12: 107, 1983.

8. Ríos, E., Pinochet, M., Mandujano, M.: "Tiempo de sangria de Ivy modificado: Valores normales en edad peđiátrica. Rev. Chil. Pediatr. 53: 552, 1982.

9. Brecher, M.: "The reproducibility and constancy of the platelet count". Am. J. Clin. Pathol. 23: 15, 1953.

10. Langdell, R.D., Wagner, R.H, Brinkhous, K.M.: "Effect of Antihemoplilic Factor on One-Stage Clotting Tests. A Presumptive Test for Hemophilia and a Simple One-Stage Antihemophilic Factor: Assay Procedure. J. Lab. Clin. Med. 41: 637, 1953. 
11. Laurell, C.B.: "Electroinmmunoassay. Stand. J, Lab. Clin. Inves1. 29 (Suppl. 124) $21,1972$.

12. Zimmerman, T.S., Hover, L. W., Dickson, L. Edgington, T.S.: "Determination of the yon Willebrand's Discase Antigen (Factor VIJ - Related Antigen) in Pla sma by Quantitative Immunoekctrophoresis". J. Lab. Clin. Mod. 86: 152, 1975.

13. Reisner, H.M. Katz, H.J., Golding, L.R., Burrow, E.S., Graham, J.B.: "Use of al simple visual atsisty of von Willebrand Factor for Diagnosis and carrier identilication". Brit. J. Haematol. 40: 339, 1978.
14. Puckham, M.A., Kinlough Rathbone, R.L., Mustard, $J, F$.: "Agaregation and Agglutination in Platelet". Function Testing. Ed. Day, H.J., Holmsen, H., Zucker, M.B. D.H.E.W. Public. N (N.I.H.) $78-1087$, Bethesda, 1978, 1. 66 .

15. Zimmerman, T.S., Roberts, J., Edgington, T.S.: "Factor VIII. Related Antigen: Multiple Molecular liorms in Human Plasma. Proc. Nat. Acad. Sci. U.S.A. 72:5121, 1975.

16. Nisson, I.M. Hoimberg. L., von Willebrand's Disease roday. Clin. Haematol \&: 147, 1979 . 